\title{
Pulmonary Infections in Patients with Rheumatoid Arthritis Who have Received Anti-TNF Therapy
}

\author{
Koichi Amano \\ Key words: rheumatoid arthritis, tumor necrosis factor, tuberculosis, pneumocystis, infliximab
}

(DOI: 10.2169/internalmedicine.45.0156)

Tumor necrosis factor (TNF) $\alpha$ is one of the major proinflammatory cytokines involved in the pathogenesis of rheumatoid arthritis (RA). In addition, TNF $\alpha$ has been revealed to develop bone destruction through activating osteoclasts. Therefore, suppression of the biological activity of TNF $\alpha$ may not only lead to improvement of clinical manifestations of RA but also to prevent joint destruction and deformity and these anti-TNF agents are considered to be the best therapeutic regimen for the management of RA patients failing to adequately respond to traditional anti-rheumatic drugs (1). In Japan there are two anti-TNF agents available for the treatment of RA. Infliximab, a chimeric mouse/human monoclonal antibody against human TNF $\alpha$ was approved in July 2003, and the other biologic agent etanercept which is a fusion protein composed of two recombinant human TNF receptor p75 molecules and the $\mathrm{Fc}$ domain of human $\mathrm{IgG}_{1}$, was introduced in March 2005. Both agents are already used worldwide, and more than 10,000 Japanese RA patients have received one of the two therapies.

On the other hand, TNF $\alpha$ plays a pivotal role in cellular immunity, especially granuloma formation in infectious diseases caused by intracellular microorganisms such as Mycobacterium tuberculosis (2, 3), Listeria monocytogenes (4), and Pneumocystis jiroveci (formerly Pneumocystis carinii) (5). Thus the use of TNF blocking agents may be associated with the increased risk of these opportunistic infections (6).

In June, 2006 issue of the journal, Imaizumi et al report two cases of opportunistic infection, extrapulmonary tuberculosis and Pneumocystis jiroveci pneumonia (PCP), which developed during infliximab therapy for RA (7).

In terms of tuberculosis in RA patients receiving TNFblockade therapy, the incidence is reported to be several times higher than that of the general population, and the ratio of extrapulmonary diseases such as miliary tuberculosis is very high (2). It is thought to be a reactivation of latent infection because most cases of tuberculosis occur within 3 months after the initiation of infliximab (2). As the authors mentioned, the post-marketing surveillance (PMS) has been done for 4,000 RA patients treated with infliximab in Japan. Eleven cases with tuberculosis were seen among the first 2,000 patients, but only two developed tuberculosis among the last 2,000 patients, most of them might have intensive screening tests for tuberculosis before starting infliximab. These PMS data revealed that infliximab therapy increased the risk of tuberculosis $(325$ per 100,000) to 13-fold that of the general population in Japan (about 25 per 100,000). In addition, 6 of $13(46 \%)$ had extrapulmonary tuberculosis, which supported the previous oversea reports $(2,3)$. The PMS data also showed that all 13 patients who suffered tuberculosis had not received isoniazid (INH) as a prophylaxis, and no patients who had taken INH developed tuberculosis. The prophylactic use of INH is effective for preventing tuberculosis and is recommended for high-risk patients (8). Since some of the high-risk patients can give a negative tuberculin skin test because of immunosuppressive treatment for RA, rheumatologists must notice early signs and symptoms of tuberculosis including extrapulmonary diseases and subsequently must take an aggressive diagnostic approach such as PCR, transbronchial biopsy. In addition, new diagnositic methods such as anti-tuberculosis glycolipid (TBGL) antibody (9) and interferon-gamma release assay may also be helpful (10).

Pneumocystis jiroveci pneumonia (PCP) is another important opportunistic infection (5). The PMS data for infliximab in Japan indicated a high incidence of PCP (15 out of 4,000 RA cases), and the median interval from the introduction of infliximab to the onset of PCP is 74 days and all of the reported PCP episodes occurred within 22 weeks after infliximab was started. These findings suggested that PCP development might be due to the reactivation of latent infection rather than to the environmental exposure, although it is said that person-to-person transmission is more likely in HIVinfected patients (11).

For early and accurate diagnosis of PCP, PCR from in- 
duced sputum is simple and easy and is superior to cytology in sensitivity, although the specificity of PCR is low because colonized Pneumocystis jiroveci can be detected (12). Serum $\beta$-D glucan value is helpful for a diagnosis of PCP (13), as in case- 2 of Imaizumi et al.

A delay in the diagnosis of opportunistic infection may increase the mortality rate in RA. Since the risk of infectious death is highest in the first 16 weeks after the initiation of anti-TNF therapy, rheumatologists should be on the lookout for opportunistic infections, particularly during the first several months after anti-TNF therapy.

\section{References}

1. Haraoui B. The anti-tumor necrosis factor agents are a major advance in the treatment of rheumatoid arthritis. J Rheumatol Suppl 72: 46-47, 2005.

2. Keane J, Gershon S, Wise RP, et al. Tuberculosis associated with infliximab, a tumor necrosis factor alpha-neutralizing agent. $\mathrm{N}$ Engl J Med 345: 1098-104, 2001.

3. Gomez-Reino JJ, Carmona L, Valverde VR, Mola EM, Montero MD. Treatment of rheumatoid arthritis with tumor necrosis factor inhibitors may predispose to significant increase in tuberculosis risk: a multicenter active-surveillance report. Arthritis Rheum 48: 2122-2127, 2003.

4. Bowie VL, Snella KA, Gopalachar AS, Bharadwaj P. Listeria meningitis associated with infliximab. Ann Pharmacother 38: 5861, 2004.

5. Tai TL, O'Rourke KP, McWeeney M, Burke CM, Sheehan K, Barry M. Pneumocystis carinii pneumonia following a second infusion of infliximab. Rheumatology 41: 951-952, 2002.

6. Cush JJ. Safety overview of new disease-modifying antirheumatic drugs. Rheum Dis Clin North Am 30: 237-255, 2004.

7. Imaizumi K, Sugishita M, Usui M, Kawabe T, Hashimoto N, Hasegawa Y. Pulmonary infectious complications associated with anti-TNF $\alpha$ therapy (Infliximab) for rheumatoid arthritis. Intern Med 45 (10): 685-688, 2006.
8. Carmona L, Gomez-Reino JJ, Rodriguez-Valverde V, et al. Effectiveness of recommendations to prevent reactivation of latent tuberculosis infection in patients treated with tumor necrosis factor antagonists. Arthritis Rheum 52: 1766-1772, 2005.

9. Maekura R, Okuda Y, Nakagawa M, et al. Clinical evaluation of anti-tuberculous glycolipid immunoglobulin $\mathrm{G}$ antibody assay for rapid serodiagnosis of pulmonary tuberculosis. J Clin Microbiol 39: 3603-3608, 2001.

10. Ferrara G, Losi M, Meacci M, et al. Routine hospital use of a new commercial whole blood interferon-gamma assay for the diagnosis of tuberculosis infection. Am J Respir Crit Care Med 172: 631$635,2005$.

11. Morris A, Beard CB, Huang L. Update on the epidemiology and transmission of Pneumocystis carinii. Microbes Infect 4: 95-103, 2002.

12. Torres J, Goldman M, Wheat LJ, et al. Diagnosis of Pneumocystis carinii pneumonia in human immunodeficiency virus-infected patients with polymerase chain reaction:a blinded comparison to standard methods. Clin Infect Dis 30: 141-145, 2000.

13. Shimizu A, Oka H, Matsuda T, Ozaki S. (1-->3)-beta-D glucan is a diagnostic and negative prognostic marker for Pneumocystis carinii pneumonia in patients with connective tissue disease. Clin Exp Rheumatol 23: 678-680, 2005.

(C) 2006 The Japanese Society of Internal Medicine http://www.naika.or.jp/imindex.html 